\title{
Sympatho-Vagal Correlates of Transient Ischemia in Ambulatory Patients
}

\author{
F Jager, GB Moody ${ }^{1}$, G. Antolič ${ }^{2}$, D. Mašič, and RG Mark ${ }^{1}$ \\ Faculty of Computer and Information Science, Ljubljana, Slovenia \\ ( ${ }^{1}$ ) Harvard-M.I.T. Division of Health Sciences and Technology, Cambridge, MA, USA \\ $\left({ }^{1}\right)$ Cardiology Division, Beth Israel Deaconess Medical Center, Boston, MA, USA \\ $\left({ }^{2}\right)$ Department of Cardiology, University Medical Center, Ljubljana, Slovenia
}

\begin{abstract}
In this paper we test the hypothesis that isolated-episode temporal patterns of ST changes result from sporadic physical activity and increased oxygen demand, and that salvo patterns are due to vascular/endothelial factors in the context of coronary vasoconstrictions or vasospasms. We used an adaptive autoregressive method to study frequencydomain sympatho-vagal correlates of neuro-autonomic control during transient ischemia for two very different groups of records in the European Society of Cardiology ST$T$ database: a group of records containing isolated $S T$ episodes, and a group of records containing salvos of $S T$ episodes. We conclude that increased sympathetic tone preceds isolated but not salvo-type ischemic episodes, and may be associated with triggering events.
\end{abstract}

\section{Introduction}

Transient ischemic ST changes (ST episodes) during ambulatory electrocardiographic monitoring most often occur in patients with stable coronary artery disease. It has been reported that $81 \%$ of the ischemic ST episodes experienced by these patients are preceded by a marked increase in heart rate [1]. Most episodes in this category appear to be due to dynamic or isometric physical exertion; the pathophysiology is increased oxygen demand, associated with increased blood presure and myocardial contractility [2]. Much less often, these patients may experience ischemic ST episodes without a prior increase in heart rate. Such episodes normally appear in association with cigarette smoking or mental stress: they occur in the context of reduced oxygen supply due to coronary vasoconstriction [2]. Ischemic ST episodes of variant (Prinzmetal's) angina due to vasospasm, unstable angina due to thrombosis, and microvascular angina (syndrome X) also belong in the latter category. The distinguishing characteris- tics of demand-driven and supply-driven ischemia, in terms of the electrocardiogram (ECG; e.g., diagnostic parameters related to heart rate and sympathovagal activity), and their interrelationships, are not yet clearly known. Differentiating between demand-driven and supply-driven ischemia on the basis of the ECG only would be of significant clinical importance, since this capability would provide an economical and noninvasive means of obtaining insights into the specific pathophysiologic mechanism responsible for ischemia in an individual patient, and thus guide in the choice of therapeutic interventions.

In our previous study [3], we described observations of distinctly different temporal patterns of ST change in the European Society of Cardiology ST$T$ Database (ESC DB) [4]: sporadic activity (isolated episodes), slow repetitive patterns (quasi-periodic episodes throughout the observation period), and salvo patterns (quasi-periodic episodes appearing in bursts). We hypothesized that (a) isolated episodes are due to increased oxygen demand resulting from sporadic physical activity, and that (b) the ST salvo episodes are due to reduced oxygen supply resulting from quasi-periodic vasoconstriction or vasospasm. Coronary blood flow is modified and regulated by the sympathetic and parasympathetic divisions of the autonomic nervous system and also by myocardial metabolic needs, and vascular/endothelial factors. While the latter factors are not easily amenable to measurements, the former can be estimated by spectral analysis of heart rate variability. Low and high frequency powers reflecting sympathetic and parasympathetic activity respectively, calculated from the Lomb periodogram of the baselinecorrected instantaneous heart rate (IHR) time series, tend to show different behavior prior to and during ischemia for the sporadic and salvo groups [5]. A considerable limitation of our previous study, however, was 
the non-stationary behavior of IHR, given the timeinvariant power spectral density estimation technique used. In this paper, we further test our hypothesis using an adaptive autoregressive (AR) method to observe frequency-domain sympatho-vagal correlates of neuro-autonomic control during transient ischemia in the ESC DB.

\section{Methods}

We classified the records of the ESC DB into groups according to temporal patterns of ST deviations [5]. For this study we chose two limited sets of records: the isolated-episode group which included 21 of the 27 records containing a single ischemic ST episode (the records excluded were duplicates from patients recorded twice, and those showing several distinct peaks in ST segment deviation during the ischemic episode, thus being likely to belong to the salvo group); and the salvo group which included 7 records each containing a salvo of 4 to 7 ischemic ST episodes.

We obtained uniformly sampled (sampling frequency of $2 \mathrm{~Hz}$ ) and smoothed IHR time series using the IPFM method [6]. From the IHR time series we measured time- and frequency-domain parameters. The latter were derived at each sample. Time series were created for the fractions of the total IHR power in the low frequency $(L F)$ band $(0.04$ to $0.15 \mathrm{~Hz})$ and in the high frequency $(H F)$ band $(0.15$ to $0.4 \mathrm{~Hz})$; and for the sympatho-vagal ratio $(L F / H F)$. For each record, we computed the mean of the time- and frequency domain parameters over the following time intervals: entire record $(R)$, the 6 minutes prior to onset of ischemia $\left(B_{6}\right)$, the 3 minutes prior to ischemia $\left(B_{3}\right)$, and during ischemia $(I)$. Aggregate average figures were derived for these values for both groups and the Student's $t$-test was used to assess the statisticall significance of differences in mean values. We also derived time trends of parameters revealing their behavior throughout the record. Among many sophisticated techniques for consistent spectral tracking of transient events in non-stationary IHR time series we chose an adaptive AR technique with a recursive least-square (RLS) algorithm [7]. The advantages of this technique are estimation of AR parameters (and power spectral density) adaptively at each sample thus tracking slow changes in the spectra, and the method's fast convergence rate. Parameters of the AR model $\mathbf{a}(n)$ are updated using following relation

$$
\mathbf{a}(n)=\mathbf{a}(n-1)-\mathbf{g}(n) e(n / n-1)
$$

where $\mathbf{g}(n)$ represents the Kalman gain vector and $e(n / n-1)$ is the estimation error. By examining the effects of model order on the spectra, we found that an
AR model of order 12 permits acceptable discrimination of the frequencies of interest. Changes in the spectra are tracked by weighting the performance index of the RLS method by a "forgetting" factor $\lambda$. One of our objectives was to track transient changes of the spectra accurately in the LF $(0.04$ to $0.15 \mathrm{~Hz})$ band. The border frequencies of this band correspond to periods of 25 and 6.7 seconds. We chose values for $\lambda$ of 0.985 , and 0.99 , corresponding to exponentially weighted estimation windows of $33(\lambda /(1-\lambda)$ samples $)$, and 49.5 seconds, respectively. (Note that the shortest annotated ischemic episode in the ESC DB is 30 seconds in length.) Narrow windows permit rapid adaptation at the cost of sensitivity to noise. By using values of $\lambda$ close to 1 (i.e., relatively long windows), the spectral estimates are made more robust, though less able to track rapid changes. Aggregate average statistics did not depend strongly on $\lambda$, while the time trends for slowly varying episodes in the isolated-episode group were smoother and more informative if using $\lambda=0.99$.

\section{Results}

Table 1 summarizes aggregate average heart rates, while table 2 shows aggregate average normalised $L F$ and $H F$ powers and the $L F / H F$ ratio for the two groups. Figures were calculated over the entire record in each case, and in windows prior to ischemic episodes and during the episodes. Table 1 shows that heart rate was significantly higher during ischemia as compared to the interval $B_{6}(p<0.05)$ in the isolated-episode group, but not in the salvo group. According to table 2 , the isolated-episode group shows a significant drop of the $L F$ power during ischemia as compared to the interval $B_{6}(p<0.01)$, slight rise of the $L F / H F$ ratio in $B_{6}$ in comparison to the interval $R$, and drop of the ratio during ischemia. The isolated-episode group significantly differs in $L F$ and $H F$ power during ischemia in comparison to the salvo group $(p<0.005, p<0.001)$, respectively, and the salvo group shows a slight rise of the $L F / H F$ ratio during ischemia as well. According to overall record statistics, we may conclude that the isolated-episode group exhibits higher heart rates. dominance of sympathetic activity (higher $L F / H F$ ratio). and lower parasympathetic activity ( $H F$ power), as compared to the salvo group. Figures 1 and 2 show the time trends of the $L F$ and $H F$ powers of the IHR and the time trend of sympatho-vagal ratio $L F / H F$ for a record from the isolated-episode and a record from the salvo group, respectively. Isolated ischemic episodes are most often preceded by an increased heart rate and a significant peak in the $L F / H F$ ratio within 10 minutes prior to the onset of ischemia (12 cases) due to the dominance of sympathetic tone (see figure 


\begin{tabular}{|c|c|c|c|c|c|}
\hline Age & Heart rate & $R$ & $B_{6}$ & $B_{3}$ & $I$ \\
\hline 54.6 & Isolated $(21)$ & 79.6 & 81.5 & 84.7 & $93.7^{*}$ \\
11.0 & std. & 11.6 & 12.6 & 14.1 & 17.5 \\
\hline 56.6 & Salvos $(7)$ & 68.2 & 69.2 & 69.8 & 72.9 \\
9.34 & std. & 13.3 & 14.1 & 14.2 & 13.5 \\
\hline
\end{tabular}

Table 1. Aggregate average heart rates as calculated throughout the records, prior to ischemia, and during ischemia (see text). Standard deviations are below. ${ }^{*} p<0.05$ versus $B_{6}$ for the isolated-episode group.

\begin{tabular}{|c|ccc|ccc|ccc|}
\hline $\mathbf{P}$ & \multicolumn{3}{|c|}{$L F$} & \multicolumn{3}{|c|}{$H F$} & \multicolumn{3}{|c|}{$L F / H F$} \\
\hline & $R$ & $B_{6}$ & $I$ & $R$ & $B_{6}$ & $I$ & $R$ & $B_{6}$ & $I$ \\
\hline Iso & 8.51 & 9.89 & $4.42_{\dagger}^{*}$ & 2.75 & 3.14 & $1.47_{\ddagger}$ & 5.69 & 6.61 & 4.81 \\
std & 4.79 & 7.59 & 4.42 & 3.01 & 4.99 & 1.86 & 2.87 & 4.42 & 3.04 \\
\hline Sal & 12.9 & 13.2 & 14.3 & 7.23 & 7.49 & 6.66 & 2.94 & 2.93 & 3.42 \\
std & 6.52 & 5.68 & 5.85 & 3.26 & 4.10 & 2.30 & 1.38 & 1.01 & 1.55 \\
\hline
\end{tabular}

Table 2. Aggregate average normalised $L F$ and $H F$ powers $\left(\left[* 10^{-2}\right]\right)$ with the $L F / H F$ ratios as calculated with $\lambda=0.985$ throughout the records, prior to ischemia, and during ischemia (see text). ${ }^{*} p<0.01$ versus $B_{6}(L F)$ for the isolated-episode group. $\dagger p<0.005$ versus $I(L F)$ for the salvo group. $\ddagger p<0.001$ versus $I(H F)$ for the salvo group.

1). In seven cases, we observed another significant peak in the $L F / H F$ ratio during the episodes, suggesting increased sympathetic outflow (see figure 1), while in six other cases the ratio dropped during the episodes, suggesting dominance of parasympathetic tone (depressor response). Prior to the episodes of salvos there is generally no change in heart rate. During the ischemia heart rate either does not change at all, or increases only after the beginning of ST change. Some episodes are accompanied by relatively small peaks in the $L F / H F$ ratio during or prior to the episodes; the others are accompanied by no obvious or explainable pattern of change in the ratio (see figure 2). It may be significant that of the six salvos in the ESC DB in which the beginning of the salvo is visible, five were preceded ( 10 to 30 minutes) by a significant peak in the $L F / H F$ ratio.

\section{Discussion and conclusions}

Ischemia is usually preceded by increases in heart rate, blood pressure, and sympathetic activity. In this setting, we would expect increased contractility, blood pressure, metabolic demand, and (consequently) ischemia, rather than local coronary vasodilation and in-

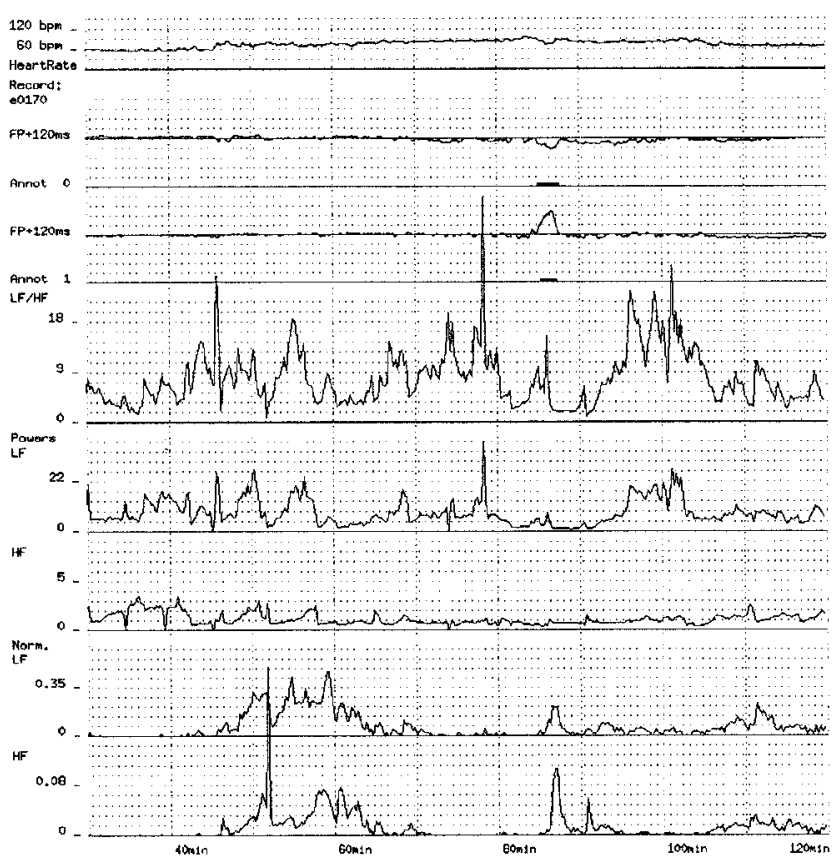

Figure 1. Time trends of the frequency-domain parameters $(\lambda=0.99)$ of the IHR for record e0170 from the isolated-episode group. From top to bottom: heart rate; ST segment deviation levels for both leads at $120 \mathrm{~ms}$ after ARISTOTLE's fiducial point (resolution: $100 \mu \mathrm{V}$ ) with the corresponding reference ischemic ST episode annotation streams; $L F / H F$ ratio; $L F$ and $H F$ powers $\left(\mathrm{bpm}^{2}\right)$, and $L F$ and $H F$ normalised powers.

creased coronary flow. Ischemia is able to elicit hemodynamic as well as neural reflexes. Moreover, ischemia also activates complex pressor (excitatory) and depressor (inhibitory) reflexes, which may alter sympathetic and parasympathetic inputs to the cardiovascular control system. Our findings regarding sympatho-vagal behavior in the isolated-episode group seem consistent with the known pathophysiology of effort angina. During severe ischemic episodes cardiac dilation may activate ventricular vagal afferents which can elicit depressor reflexes. During less severe episodes, sympathetic sensors may stimulate pressor reflexes [8].

The pathophysiology of ischemia preceded not by an increase in heart rate, but by mental stress and elevated blood pressure, is more difficult to describe. If (as we hypothesize) salvos of ischemic episodes are triggered by salvos of vasospasms, we may further conjecture that quasi-periodic platelet adhesion and break-off may account for the recurrent vasospasms. In this case, we would not necessarily expect an increase in sympathetic tone prior to a salvo of ischemic episodes.

A recent study suggests that parasympathetic withdrawal may often be a component of the mechanism 


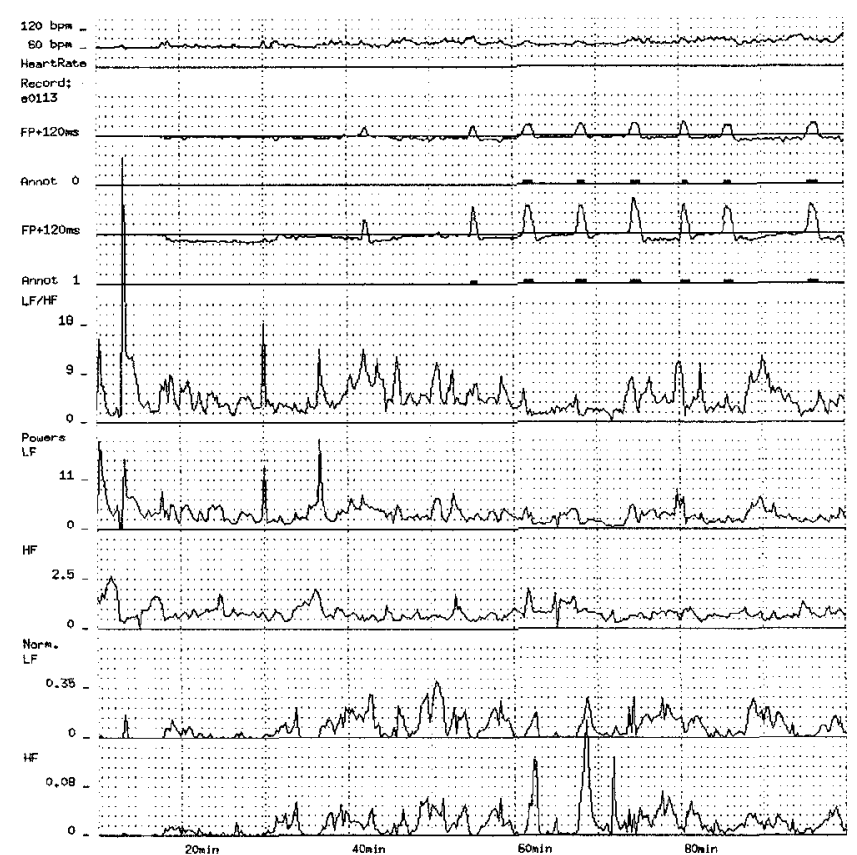

Figure 2. Time trends of the frequency-domain parameters $(\lambda=0.985)$ of the IHR for the record e0113 from the salvo group. (For legend see caption to figure 1.)

leading to spontaneous coronary vasospasms [9]. In that study of patients with typical variant angina and anginal pain, $H F$ frequency reduction before ST segment elevation with a return to basal levels at peak ST elevation, and increase of $L F$ frequency at peak ST elevation, were observed in episodes of transmural ischemia. Our findings with respect to individual episodes within salvos only partially support those of [9]; we observe, however, that $L F$ power increases only slightly, but not significantly, during the episodes. We also observe a peak in the $L F / H F$ ratio prior to the salvos.

Apart from neurogenic factors, local endothelial factors (and medications to a certain extent) play a major role in regulating and modifying blood flow in acute spastic and microvascular angina [10]. Both neurogenic and endothelial factors regulate the diameter of the coronary vessels and may result in modified or even paradoxical response. Thus, if salvos of ischemic episodes are caused by vasospasm, it may be inappropriate to describe the pathophysiology of salvo group in terms of sympatho-vagal influences alone.

Other limitations of the present study are small number of salvo records and the 2-hour duration of the records of the ESC DB, thus leaving open questions regarding the temporal ST deviation patterns over longer observation periods.

We conclude that slightly, but not significantly, in- creased sympathetic tone precedes isolated episodes but not individual salvo type episodes. Changes in autonomic neural tone are likely to be associated with triggering events. Autonomic responses during ischernic episodes are variable.

\section{Acknowledgment}

This publication is based on work sponsored by the U.S.Slovenian Science and Technology Joint Fund in cooperation with the Department of Health and Human Services, U.S.A. and the Ministry for Science and Technology of the Republic of Slovenia under Project number 95-158.

\section{References}

[1] Andrews TC, Fenton T, Toyosaki N, Glasser SP, Young PM, MacCallum G, Gibson RS, Shook TL, Stone PH. Subsets of ambulatory myocardial ischemia based on heart rate activity (circadian distribution and response to anti-ischemic medication). Circulation 88:92-100 (1993).

[2] Andrews TC, Stone PH. Recent development in the understanding and management of angina pectoris in patient with stable coronary artery disease. Current Opinion in Cardiology 6:503-510 (1991).

[3] Jager F, Moody G, Mark RG. Characterization of transient ischemic and non-ischemic ST segment changes. Computers in Cardiology 1995; 721-724.

[4] Taddei A, Distante G, Emdin M, Pisani P, Moody GB, Zeelenberg C, Marchesi C. The European ST$T$ database: standard for evaluating systems for the analysis of ST-T changes in ambulatory electrocardiography. Eur. Heart J. 13:1164-1172 (1992).

[5] Jager F, Moody G, Pavlič B, Blažina I, Župič I, Mark $R G$. Characterization of temporal patterns of transient ischemic ST change episodes during ambulatory ECG monitoring. Computers in Cardiology 1996; 681-684.

[6] DeBoer RW, Karemaker JM, Strackee J. Comparing spectra of a series of point events particularly for the heart rate variability data. IEEE Trans Biomed End 31(4):384-387 (1984).

[7] Akay M. Biomedical signal processing. Academic Press, Inc., 1994.

[8] Kulbertus HE, Franck G, editors. Neurocardiology. Futura Publishing Company, Inc., 1988.

[9] Lanza GA, Pedrotti P, Pasceri V, Lucente M, Crea F, Maseri A. Autonomic changes associated with spontaneous coronary spasm in patients with variant angina. $J$ Am Coll Cardiol 28:1249-56 (1996).

[10] Bertolet BD, Pepine CJ. The vascuar endothelium as a key to understanding coronary spasm and syndrome X. Current Opinion in Cardiology 6:496-502 (1991).

Address for correspondence:

Franc Jager

Faculty of Computer and Information Science,

Tržaška 25, 1001 Ljubljana, Slovenia

Internet: franc@manca.fri.uni-lj.si 